\title{
Medulloblastoma, Non-WNT/Non-SHH, Group 3
}

National Cancer Institute

\section{Source}

National Cancer Institute. Medulloblastoma, Non-WNT/Non-SHH, Group 3. NCI

Thesaurus. Code C129445.

Medulloblastoma not associated with activation of the WNT pathway or sonic hedgehog (SHH) pathway. MYC amplifications may be present. TP53 mutations are absent.

Patients in this group are usually young children. The overall survival is the worst among all the molecular groups. 